\title{
The pymolsnips library to aid the writing of PyMOL scripts
}

\author{
B Mooers ${ }^{1}$, M Brown $^{2}$ \\ ${ }^{1}$ Universtiy of Oklahoma Health Sciences Center, Oklahoma City, OK, ${ }^{2}$ University of Oklahoma \\ Health Sciences Center, Oklahoma City, OK \\ blaine-mooers@ouhsc.edu
}

Scripts of PyMOL commands are useful for applying the numerous parameters required to exert exquisite control over the appearance of a molecular model. Unfortunately, the vast majority of PyMOL users have trouble recalling the commands and their syntax in the PyMOL scripting language because they do not use PyMOL everyday. This difficulty with the recall of commands slows the writing of new scripts. Experienced users address this problem by recycling their old script files, but this option does not work well when embarking on a new task for which a similar script cannot be found. A possible solution is to build up a new script by re-using code fragments for small parts of the task at hand. The code fragments can be accessed from a library of code fragments while writing the script from inside a text editor that supports snippet libraries. We developed such a library of code fragments--or snippets--for 20 popular text editors including the very popular Visual Studio Code, Atom, Sublime Text, vim, emacs, and Jupyter Notebook. We have included lexers where needed for syntax highlighting with colored text. This library is available on GitHub with instructions for installation on the Mac, Windows, and Linux operating systems. We expect that the library will lower motivational barriers to writing scripts to make figures in PyMOL and thereby help many PyMOL users become more productive at using PyMOL to do structural analyses and generate figures for publication.

Acta Cryst. (2020). A76, a75 